\title{
Energy loss in heavy ion collisions
}

\author{
Mikhail Tokarev ${ }^{1 *}$, Imrich Zborovský ${ }^{2}$ \\ ${ }^{1}$ Joint Institute for Nuclear Research, 141980 Dubna, Russia \\ 2 Nuclear Physics Institute, Academy of Sciences of the Czech Republic, \\ 25068 Rež, Czech Republic
}

DOI: http://dx.doi.org/10.5689/UA-PROC-2010-09/41

\begin{abstract}
Energy loss of particles created in heavy ion collisions characterizes properties of the nuclear medium. Dependence of the energy loss on the momentum of the produced hadron, energy and centrality of the collision is studied in a microscopic scenario of constituent interactions in the nuclear environment. Experimental data on inclusive spectra measured in heavy ion collisions at RHIC over a wide range of the energy $\sqrt{s_{N N}}=9-200 \mathrm{GeV}$ are analyzed in the framework of the proposed scenario.
\end{abstract}

\section{Introduction}

The measurements of particle spectra at the Relativistic Heavy Ion Collider (RHIC) led to the discovery of a substantial suppression of hadron yields in nucleus-nucleus collisions relative to proton-proton data $[1,2,3,4]$. The suppression is observed in the region of high transverse momenta, typically more than a few $\mathrm{GeV} / \mathrm{c}$. It is connected with the energy radiations of the outgoing high- $p_{T}$ partons propagating through dense matter formed in the central collisions of heavy nuclei. The energy loss in the dense medium is substantially larger than in the vacuum. As noted in [5], this can essentially modify the behavior of the system near a critical point. In dependence on collision energy, the energy loss can differently smear signals which could indicate to characteristic changes in the thermodynamic properties of the system. Therefore the Energy Scan Programm carried out at SPS [6] and RHIC [7] is of great importance to search for and study of signatures of phase transition and critical point of nuclear matter produced in heavy ion collisions. In the contribution we use the $z$-scaling approach for estimation of the energy loss as a function of the collision energy, transverse momentum and centrality.

\section{$2 \quad z$-Scaling}

A regularity of hadron production in high energy proton-(anti)proton collisions, known as zscaling, has been established in papers $[8,9]$. It manifests itself in the fact that the inclusive spectra of various types of hadrons can be described by a universal scaling function. It holds over a wide range of transverse momenta, registration angles, collision energies and secondary particle multiplicities. The scaling function $\psi(z)$ depends on a single scaling variable

$$
z=z_{0} \Omega^{-1}
$$

* Speaker 
where

$$
z_{0}=\frac{\sqrt{s_{\perp}}}{\left(d N_{c h} /\left.d \eta\right|_{0}\right)^{c} m}
$$

and

$$
\Omega\left(x_{1}, x_{2}, y_{a}, y_{b}\right)=\left(1-x_{1}\right)^{\delta_{1}}\left(1-x_{2}\right)^{\delta_{2}}\left(1-y_{a}\right)^{\epsilon}\left(1-y_{b}\right)^{\epsilon}
$$

are functions of kinematic quantities. The value of $z_{0}$ is proportional to the transverse kinetic energy $\sqrt{s_{\perp}}$ of the constituent binary subprocess required for production of an inclusive particle $\left(m_{1}\right)$ and its recoil partner $\left(m_{2}\right)$. The parameter $c$, which has meaning of the specific heat of the produced medium, determines the functional dependence of $z_{0}$ on the multiplicity density $d N_{c h} /\left.d \eta\right|_{0}$ of charged particles in the central rapidity region $\eta=0$. The constant $m$ is fixed at the value of the nucleon mass. The quantity $\Omega$ is interpreted as a relative number of the constituent configurations which include binary subprocesses corresponding to the fractions $x_{1}$ and $x_{2}$ of colliding hadrons momenta and to the momentum fractions $y_{a}$ and $y_{b}$ of the secondary objects produced in these subprocesses. The selected binary subprocess, in terms of which the variable $z$ is defined, is determined by the maximum of $\Omega\left(x_{1}, x_{2}, y_{a}, y_{b}\right)$ with the kinematic constraint

$$
\left(x_{1} P_{1}+x_{2} P_{2}-p / y_{a}\right)^{2}=M_{X}^{2} .
$$

The mass of the recoil system in the production of an inclusive particle with the momentum $p$ is written in the form

$$
M_{X}=x_{1} M_{1}+x_{2} M_{2}+m_{2} / y_{b}
$$

Equation (4) accounts for the locality of the constituent interaction and sets a kinematic restriction on the momentum fractions $x_{1}, x_{2}, y_{a}$, and $y_{b}$.

The variable $z$ possesses the property of a fractal measure. It diverges in a power like manner with the increasing resolution relative to the constituent subprocesses $\left(x_{1}, x_{2}, y_{a}, y_{b} \rightarrow 1\right)$. The parameters $\delta_{1}$ and $\delta_{2}$ are corresponding fractal dimensions of the colliding objects. For nuclear collisions we set $\delta_{1,2}=A_{1,2} \delta$ with $\delta$ being the nucleon fractal dimension. The parameter $\epsilon$ stands for a fractal dimension of the fragmentation process. The parameter $c$ is interpreted as a "specific heat" of the medium associated with production of the inclusive particle. For $p p$ collisions the parameters $\delta, \epsilon$, and $c$ were found to be independent of kinematic variables over a wide energy range $\sqrt{s}=19-200 \mathrm{GeV}$. The determination of the fractions $x_{1}, x_{2}, y_{a}, y_{b}$ corresponding to the selected binary subprocess allowed us to develop a microscopic scenario of the interaction at a constituent level. The dependencies of the momentum fractions and the recoil mass $M_{X}$ on the transverse momentum $p_{T}$ of the inclusive particle, as well as on the energy and centrality of the collisions represent important features of the interaction.

The scaling function $\psi(z)$ is expressed in terms of experimentally measurable inclusive cross section for the reaction $P_{1}+P_{2} \rightarrow p+X$. It can be written in the form

$$
\psi(z)=-\frac{\pi s}{(d N / d \eta) \sigma_{\text {inel }}} J^{-1} E \frac{d^{3} \sigma}{d p^{3}},
$$

where $\sigma_{i n}$ is the total inelastic cross section, $d N / d \eta$ is the multiplicity density of registered particles and $J$ is the Jacobian for the transformation from $\left\{p_{T}^{2}, y\right\}$ to $\{z, \eta\}$. The normalization

$$
\int_{0}^{\infty} \psi(z) d z=1
$$

allows us to interpret $\psi(z)$ as the probability density of production of the inclusive particle with the corresponding value of the variable $z$. 


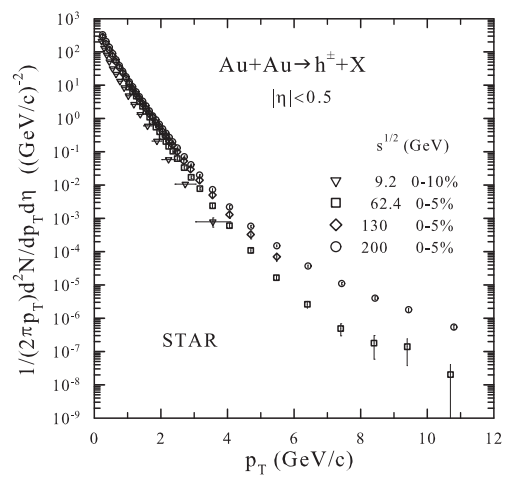

a)

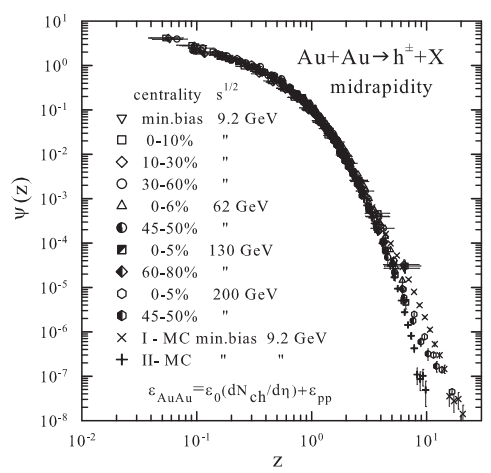

b)

Figure 1: (a) The transverse momentum spectra of charged hadrons [1, 10, 11, 12] produced in central $A u A u$ collisions at $\sqrt{s_{N N}}=9.2,62.4,130,200 \mathrm{GeV}$, and $|\eta|<0.5$. (b) The scaling function $\psi(z)$. MC simulations for I and II scenarios at $\sqrt{s_{N N}}=9.2 \mathrm{GeV}$.

\section{$3 \quad$ Energy loss in $A A$}

In this section we use the microscopic scenario of particle production based on the $z$-scaling for estimation of the energy loss of the produced particles in nuclear medium. In the framework of such scenario, the energy loss during formation of the inclusive particle is characterized by the momentum fraction $y_{a}$. The value of $y_{a}$ depends on the fragmentation dimension $\epsilon$. The recoil object moving in the away side of the inclusive particle is characterized by the recoil mass $M_{X}$. Its value depends at high energies predominantly on the momentum fraction $y_{b}$.

Figure 1(a) shows $p_{T}$-dependence of the spectra of charged hadrons $[1,10,11,12]$ produced in the central $A u A u$ collisions at $\sqrt{s_{N N}}=9.2,62.4,130$, and $200 \mathrm{GeV}$ in central rapidity range $|\eta|<$ 0.5 . The spectra cover a wide range of the transverse momentum $\left(p_{T}=0.35-10.8 \mathrm{GeV} / \mathrm{c}\right)$ with change of more than eight orders of magnitude. Figure 1(b) demonstrates the $z$-presentation of hadron yields. Data for the central and peripheral $A u A u$ collisions $[1,4,10]$ at $\sqrt{s_{N N}}=$ $62.4,130,200 \mathrm{GeV}$, and the central $(0-10 \%)$, mid-central $(10-30 \%)$, peripheral $(30-60 \%)$ and minimum bias $A u A u$ collisions [12] at $\sqrt{s_{N N}}=9.2 \mathrm{GeV}$ are shown. The centralities are characterized by different multiplicity densities $d N_{\mathrm{ch}} /\left.d \eta\right|_{0}$ of charged particles produced at midrapidity.

The independence of $\psi(z)$ on the collision energy for $\sqrt{s_{N N}}=62.4,130,200 \mathrm{GeV}$ in peripheral $A u A u$ collisions is consistent with the constant value of the fractal dimension $\delta_{A u A u}=197 \cdot \delta$ and the same value of $\delta=0.5$ as in $p p$ interactions. Independence of $\psi(z)$ on centrality for $A u A u$ interactions can be achieved at these energies by the constant value of the "specific heat" $c_{A u A u}=0.11$, provided multiplicity dependence of the fragmentation dimension $\epsilon_{A A}$ in the form

$$
\epsilon_{A A}=\epsilon_{0}\left(d N_{\mathrm{ch}}^{A A} / d \eta\right)+\epsilon_{p p}
$$

The value of $\epsilon_{p p}=0.2$ was obtained from analysis of charged hadrons for $p p$ collisions [8, 9]. Allowing the coefficient $\epsilon_{0}$ to be dependent on the collision energy, one can obtain the identical $\psi(z)$ for $p p$ and $A u A u$ interactions for all centralities in the considered energy range. 


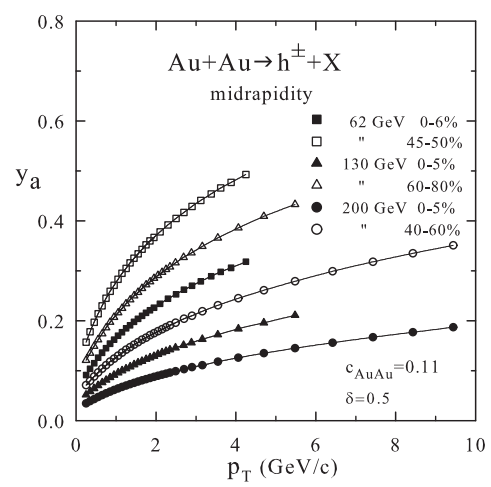

a)

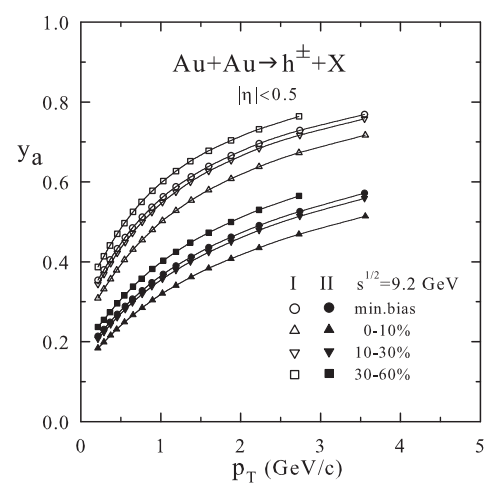

b)

Figure 2: The dependence of the fraction $y_{a}$ on the transverse momentum $p_{T}$ for charged hadrons produced in $A u A u$ collisions in midrapidity range at different energies $\sqrt{s_{N N}}=62.4$, 130, $200 \mathrm{GeV}$ (a), $9.2 \mathrm{GeV}$ (b), and centralities. The open and closed symbols in (b) correspond to I and II scenarios of hadron production, respectively.

The increase of $\epsilon_{A A}$ with the multiplicity density (centrality) is connected with a decrease of the momentum fraction $y_{a}$. This corresponds to larger energy loss by the formation of the inclusive hadron. On the other hand, the energy loss depends on the traversed medium which converts it into the multiplicity of the associated particles. The larger $\epsilon_{A A}$ the more energy loss consumed to production of secondary particles. In a such way the produced medium is via the multiplicity density characterized by the amount of the energy loss.

A correlation between $c_{A u A u}$ and $\delta$ for $A u A u$ collisions at $\sqrt{s_{N N}}=9.2 \mathrm{GeV}$ was found. The restoration of the universal shape of $\psi(z)$ in the measured $p_{T}$ range at this energy can be reached in two scenarios: I $-c_{A u A u}=0.23, \delta=0.5$ and II $-c_{A u A u}=0.11, \delta=0.15$. The first one (illustrated with data in Fig.1(b)) corresponds to the large and the second one to the small (energy independent) value of the "specific heat" c. The measured $p_{T}$ range and large errors at high $p_{T}$ do not allow to discriminate between both scenarios. Measurement of hadron distributions at $\sqrt{s_{N N}}=9.2 \mathrm{GeV}$ versus centrality for $p_{T}>4 \mathrm{GeV} / \mathrm{c}$ is desirable to resolve the problem and study the dependence of the energy loss in this region. The Monte Carlo UrQMD [13] simulations for minimum bias events indicate large differences between both scenarios in the corresponding high- $z$ region (see Fig.1(b)).

Figure 2(a) shows the dependence of the fraction $y_{a}$ on the transverse momentum of charged hadrons produced in the central and peripheral $A u A u$ collisions at $\sqrt{s_{N N}}=62.4,130,200 \mathrm{GeV}$ in midrapidity range. Energy loss by the formation of the inclusive particle is proportional to $\left(1-y_{a}\right)$. As seen from Fig.2(a), the energy loss decreases with the increasing momentum $p_{T}$ and increases with the collision energy and centrality. Similar trends (Fig.2(b)) are seen at lower energy $\sqrt{s_{N N}}=9.2 \mathrm{GeV}$ for both scenarios. The energy loss is smaller for the scenario I (open symbols) in comparison with the scenario II (closed symbols). The energy loss in the central $A u A u$ collisions at $p_{T}=4 \mathrm{GeV} / \mathrm{c}$ was found (Fig.2(a)) to be about $70 \%, 82 \%$, and $87 \%$ at $\sqrt{s_{N N}}=62.4,130$, and $200 \mathrm{GeV}$, respectively. At $\sqrt{s_{N N}}=9.2 \mathrm{GeV}$ and $p_{T}=4 \mathrm{GeV} / \mathrm{c}$ the energy loss in the central collisions is estimated (Fig.2(b)) to be about $30 \%$ and $50 \%$ for the scenarios I and II, respectively. 


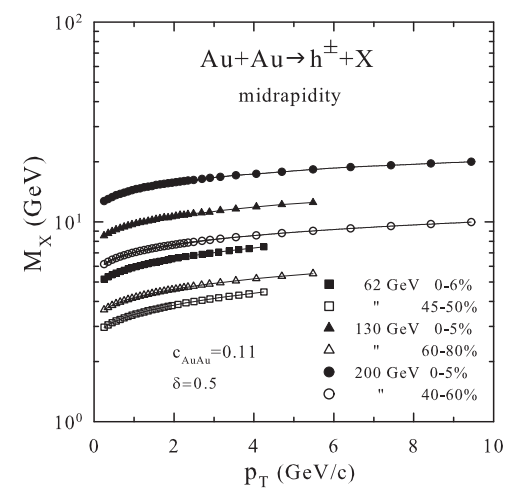

a)

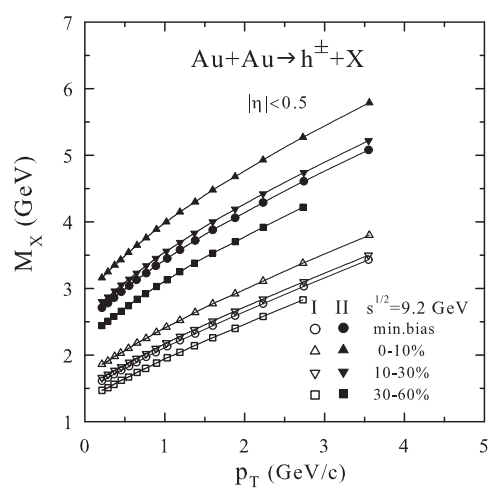

b)

Figure 3: The dependence of the recoil mass $M_{X}$ on the transverse momentum $p_{T}$ for charged hadrons produced in $A u A u$ collisions in midrapidity range at different energies $\sqrt{s_{N N}}=62.4$, 130, $200 \mathrm{GeV}$ (a), $9.2 \mathrm{GeV}$ (b), and different centralities. The open and closed symbols in (b) correspond to I and II scenarios of hadron production, respectively.

The recoil mass $M_{X}$ in the subprocess is associated with the production of the inclusive particle. It depends on the momentum fractions $x_{1}$ and $x_{2}$ of the interacting objects with the masses $M_{1}$ and $M_{2}$. The dependence of $M_{X}$ on $y_{a}$ and $y_{b}$ is determined by (5) with $x_{1,2}=x_{1,2}\left(y_{a}, y_{b}\right)$ given in [8]. This includes the dependence of $M_{X}$ on the fractal dimensions $\delta_{1}, \delta_{2}$, and $\epsilon$. The recoil mass reflects therefore an internal connection to the structure of the colliding objects, constituent interactions, and process of formation of the individual hadrons.

Figure $3(\mathrm{a})$ shows the dependence of $M_{X}$ on the transverse momentum of charged hadrons produced in the central and peripheral $A u A u$ collisions at $\sqrt{s_{N N}}=62.4,130$, and $200 \mathrm{GeV}$ in midrapidity range. The similar dependencies of $M_{X}$ on $p_{T}$ at $\sqrt{s_{N N}}=9.2 \mathrm{GeV}$ for the scenarios I and II are depicted in Fig.3(b). All curves demonstrate growth with $p_{T}$ which is followed at $\sqrt{s_{N N}}=62.4,130$, and $200 \mathrm{GeV}$ by a successive flattening. The dependencies reveal a characteristic increase with the collision energy. As seen, the values of $M_{X}$ become larger with the collision centrality. This means that the momentum balance in a subprocess underlying the hadron production is compensated with growing number of particles moving in the away side direction when the centrality of the nuclear collisions increases. Figure 3(b) shows a sharp growth of $M_{X}$ with $p_{T}$ at $\sqrt{s_{N N}}=9.2 \mathrm{GeV}$ in the range $p_{T}=0.3-4 \mathrm{GeV} / \mathrm{c}$ without any indication on a flattening. The scenario with large specific heat (open symbols) corresponds to smaller values of $M_{X}$ in comparison with the scenario II (full symbols). Consequently, the large specific heat scenario at $\sqrt{s_{N N}}=9.2 \mathrm{GeV}$ would reflect the production of more compact subsystems in the constituent interactions with smaller energy loss.

\section{Conclusion}

Experimental data on inclusive spectra of charged hadrons produced in $A u A u$ collisions at RHIC energies $\sqrt{s_{N N}}=9.2,62.4,130$, and $200 \mathrm{GeV}$ were analyzed in the framework of $z$ scaling. The experimental data in $z$-presentation indicate similarity as a characteristic feature 
of mechanism of hadron production. This property includes structure of the colliding objects, interaction of their constituents, and character of the fragmentation process. A microscopic scenario of nucleus-nucleus interactions at a constituent level in terms of momentum fractions was used to estimate the energy loss of the secondary partons passing through the medium created in heavy ion collisions. It was shown that energy loss increases with the collision energy and centrality, and decreases with $p_{T}$.

It was demonstrated that the universality of the shape of $\psi(z)$ of hadron production in $A u A u$ collisions at $\sqrt{s_{N N}}=62.4,130,200 \mathrm{GeV}$ can be preserved for the constant value of the "specific heat" $c_{A u A u}=0.11$ for all centralities, provided the fragmentation dimension $\epsilon_{A u A u}$ increases with multiplicity. A correlation between the parameters $c_{A u A u}$ and $\delta$ with change of their values at lower energy $\sqrt{s_{N N}}=9.2 \mathrm{GeV}$ was found. Two scenarios (with large and small "specific heat") of hadron production at this energy were suggested. A preferable kinematic region to discriminate between both scenarios corresponds to measurements of spectra at higher transverse momentum $p_{T}>4 \mathrm{GeV} / \mathrm{c}$.

The obtained results may be exploited to search for phase transition, critical point, and study of new physics phenomena in hadron production in heavy ion collisions.

\section{$5 \quad$ Acknowledgments}

The investigations have been supported by the IRP AVOZ10480505, by the Ministry of Education, Youth and Sports of the Czech Republic grants LA08002, LA08015. and by the special program of the Ministry of Science and Education of the Russian Federation, grant RNP.2.1.1.2512.

\section{References}

[1] J. Adams et al. [STAR Collababortaion], Phys. Rev. Lett. 91 (2003) 172302.

[2] I. Arsene et al. [BRAHMS Collaboration], Phys. Rev. Lett. 91 (2003) 072305.

[3] S. S. Adler et al. [PHENIX Collaboration], Phys. Rev. C 69 (2004) 034910.

[4] B. B. Back et al. [PHOBOS Collaboration], Phys. Rev. Lett. 94 (2005) 082304.

[5] M. V. Tokarev and I. Zborovský, Phys. Part. Nucl. Lett. 7 (3) (2010) 160.

[6] M. Gazdzicki [for the NA61/SHINE Collaboration], J. Phys. G: Nucl. Part. Phys. 36 (2009) 064039.

[7] H. Caines [for the STAR Collaboration], arXiv:0906.0305v1.

B. I. Abelev et al. [STAR Collaboration], SN0493: Experimental Study of the QCD Phase Diagram and Search for the Critical Point: Selected Arguments for the Run-10 Beam Energy Scan, http://drupal.star.bnl.gov/STAR/starnotes/public/sn0493.

B. I. Abelev et al. [STAR Collaboration], Phys. Rev. C 81 (2010) 024911.

[8] I. Zborovský and M. V. Tokarev, Phys. Rev. D 75 (2007) 094008.

[9] I. Zborovský and M. V. Tokarev, Int. J. Mod. Phys. A 24 (2009) 1417.

[10] C. Adler et al. [STAR Collababortaion], Phys. Rev. Lett. 89 (2002) 202301.

[11] C. A. Gagliardi [for the STAR Collaboration], Eur. Phys. J. C 43 (2005) 263.

[12] M. V. Tokarev [for the STAR Collaboration], arXiv:nucl-ex/1004.5582.

[13] UrQMD Monte Carlo code, http://urqmd.org. 